\title{
Mechanizing cellular automata
}

\section{Whether or not it is possible to represent hydrodynamical systems by autonomous entities on the vertices of a lattice threatens to become an unreal issue.}

THE news this week (see p. 103) of the new parallel architecture computer developed by Thinking Machines of Cambridge, Massachusetts, should be read in conjunction with two unusually contradictory papers in Physical Review Letters for 15 April. Both are stimulated by an as yet unpublished paper by J. Salem and Stephen Wolfram.

Wolfram is the Princeton-based physicist who carries a torch for von Neumann's model of the cellular automaton, the microscopic entity in an array whose state at any time is determined by its own state at some previous time and by those of its immediate neighbours.

Steven A. Orszag and Victor Yakhot first set out to show that cellular automata cannot be adapted in the real world to solve the problems of fluid dynamics (ibid., 56, 1691; 1986). The next paper (Margolus, N., Toffoli, T. and Vichniac, G., ibid., 56, 1694; 1986), based on the same unpublished paper, purports to reach the opposite conclusion. What is the innocent reader to conclude?

There is nothing startlingly novel about the model of the cellular automaton. Fill accessible space with microscopic cells, most simply of the same size. Then let each be occupied (or empty of) a physical entity which can be in only one of several states (most simply, finite in number), and then allow that future generations of this system will evolve, with the passage of time, according to rules applying equally to all of them (see Wolfram, S., Nature 311, 419; 1984).

Such a system can obviously be a model for various aspects of the real world. If the entities occupying separate cells are members of a population, the evolution of the arrangement as time goes on may be a model of how a living population evolves. But if the cells are occupied by tiny magnets allowed to point in one direction or the opposite (for example), the system is a model for a ferromagnet.

Such systems are also capable of modelling the behaviour of ordinary dynamic systems such as those forever cropping up in physics. Anybody who doubts that has merely to recall that systems of differential equations can be approximated to by finite difference equations which may be more conveniently soluble, but which are in any case a convenient way of writing a computer program to simulate the original set of equations.

So why not think of using networks of cellular automata as means of solving real problems in applied physics? A few simple systems are easily imagined. Suppose, for example, that the objective is to follow the time course of a plucked violin string or of a bent beam; one might approximate the system by a one-dimensional array of cellular automata whose individual states might represent the displacement of the string from a reference datum. If the evolution of the system is then described simply in terms of the instantaneous state of each element and its nearest neighbours, the approximation is certainly no worse than that familiar in the simple textbooks of applied mathematics, where only first and second derivatives seem to matter.

The argument that has now arisen stems from what must be the most ambitious goal of all, how to use networks of cellular automata to stimulate the motion of fluids with real thermodynamic properties, heat capacity, vorticity and friction, for example. This, it seems, is what Salem and Wolfram have been up to, in a paper with the title "Thermodynamics and Hydrodynamics with Cellular Automata", which it is said is "to be published".

Orszag and Yakhot are against the conclusions of that unpublished paper, Margolus and his colleagues show that the arguments are valid. A curious almostethical point arises at this stage. It is rare that journals publish disputatious accounts of a paper that has not yet been made accessible to readers. The issue is only nearly ethical; readers who do not care for having to read between the lines can always read some other journal.

Reading between the lines, what Salem and Wolfram have done is to argue that it should be possible to represent a real thermodynamic fluid by a system of cellular automata in the sense that the space and time averages of the properties of cellular automata will follow the behaviour of incompressible fluids which are described by the Navier-Stokes equations. Friction enters, producing heat (and robbing the system of kinetic energy).

A crucial part of the Salem and Wolfram argument (again between the lines) is that the coming of computers with parallel architecture should make these simulations more accurate and also more real.

Orszag and Yakhot do not dispute the virtues of computers made from, say, 64,000 microprocessors strung together (who could?). Their quarrel is with the underlying physics and the computational economy of the cellular automata models. Reading between the lines, one can conclude that Salem and Wolfram represent continuous variables such as the velocity (of the fluid) in a velocity field by means of averages over groups of neighbouring cells carrying separate cellular automata. Questions then arise about the efficacy of the representation; either there are so few cells in the group over which average values represent dynamical variables in the real world that the calculation is inaccurate, or the group is large enough to be realistic but the computational work is enormously increased. The essence of the argument is that tractable networks of cellular automata will usually be unrealistic, but that realistic networks will be less easily soluble than the equations to which they are an approximation.

Margolus et al. write in a quite different vein. "Surprisingly enough, cellular automata can faithfully model continuum systems such a fluids ... [but] are poorly supported by conventional scientific computers ... more appropriate architecture can easily gain a performance factor of at least 10,000 . . ." Part of the trick (to be revealed in the unpublished paper by Salem and Wolfram) is to devise the generation rules for the automata so that microscopic reversibility is assured. Margolus and his colleagues show, among other things, some pretty diagrams in which a central blob of fluid on a twodimensional plane spreads outwards in a growing concentric ring. The authors surprisingly boast that one simple system (a two-dimensional lattice gas) is macroscopically as well as microscopically reversible.

Quite what the dispute is about can be read only between the lines, but there is an obvious and simple resolution of it: simply build computer systems with as many microprocessors as there are atoms whose behaviour must be simulated, and make sure that they respond at least as quickly. Then the objections of the Orszags and Yakhots of this world will melt away. As it happens, Margolus and his colleagues say that it should be possible to deal with $10^{16}$ elements (the square of the cube root of Avogadro's number) within a decade, and to "span the gap between the microscopic and the macroscopic world" soon afterwards. That may be possible, but the question will remain of whether it is science.

John Maddox 